\title{
Study on Correlation between Electrical Parameters and Texture Parameters of Lingwu Long-Jujube during Storage Process
}

\author{
Zhou shiping, Zhang haihong*, Zhang zhong, Ma qihu, Ma Xuelian, Wang Huiqian \\ Agricultural College, Ningxia University, Yinchuan 750021, China
}

\begin{abstract}
In order to study the correlation between electrical parameters and texture parameters, the electrical parameters of Ling-wu long-Jujube picked and stored at room temperature were measured dynamically at 25 frequency points in the scope of $3.16 \mathrm{kHz}$ to $50.12 \mathrm{kHz}$ with HIOKI 3532-50 LCR, and the sensitive electrical parameters and appropriate test frequency were screened out. Texture parameters such as pericarp strength, brittleness, pulp firmness, and hardness were measured by puncture test. The regression equation was established according to the correlation between electrical parameters and texture parameters. The results showed that the sensitive parameters of the complex impedance $Z$, inductance Lp and reactance $X$ decreased in exponential form as the test frequency increasing; under the same frequency, $\mathrm{Z}, \mathrm{Lp}$ and $\mathrm{X}$ increased as the storage prolonged. With its aging, jujube hardness, brittleness and pulp firmness decreased gradually while pericarp strength increased. Under the appropriate $3.16 \mathrm{kHz}$ testing frequency, electrical parameters and texture parameters have high correlation. The regression equations of hardness, brittleness, and pulp firmness were established, and their correlation with electrical parameters reached significant level $(\mathrm{P}<0.05)$. Nondestructive testing of long jujube texture quality based on its electrical parameters is feasible.

KEYWORD: long-jujube; electrical parameters; texture parameters; correlation analysis
\end{abstract}

\section{INTRODUCTION}

Ling-wu long-Jujube is one of the most distinctive jujubes in China. It has big size, bright color, thin pericarp and, crisp texture, delicious taste, rich nutrition, and high quality identification. Its marketing mode is mainly sales of fresh jujubes. Texture indicators such as hardness, brittleness are very important in long jujube quality measuring (Feng,2011). Long jujube texture is mainly determined by the factors such as pericarp parenchymal cell sizes, quantity, close degrees of cell arrangement, cell wall thickness, and the difference of pulp cells in inside and outside layers. Long jujube texture properties have close relations with its biochemical changes. Along with ripening, its pericarp cell wall components are degraded by enzymes. Parenchymal cell structure changes and causes the fruit texture changes. The change of long jujube texture can directly affect its quality. The fruit texture testing with physical property analyzer is a new type of mechanical testing methods developed in recent years (Yaping, Hongbo, 2010). It is more accurate and objective than sense evaluation, and can truly reflect fruit quality changes and preservation effects. It not only can avoid the interference caused by human factors, overcome the shortcoming of traditional sense evaluation, but also well reflect the rheology characteristics of the whole fruit, and obtain the parameters such as the pericarp strength, brittleness, pulp hardness. The data are precise and the evaluation is more objective. However, fruit texture testing with physical property analyzer is a destructive test (Camps et al., 2005\& Qinghua et al., 2011); it is more clearly meaningful to look for a reliable, convenient and rapid nondestructive detection method for predicting and evaluating the jujube mechanical quality in storage and transport.

Lingwu long-Jujube is a kind of dielectrics. In the microcosmic point of view, an electric field exists in jujube internal. It changes its location within the scope of the molecules and then causes the radical changes of the electric field intensity. The microscopic features make jujube show the unique dielectric, conductive, and bioelectric properties. It also determines the physical (hardness and crispness) and physiological (maturity, freshness) and chemical (water, sugar and acidity) characteristics of Lingwu Long-Jujube. Electrical characteristics based fruit nondestructive testing technology received wide attentions in recent years. Mainly based on fruit electrical parameters such as dielectric constants, impedance and resistance, fruit nondestructive 
testing technology measures fruit quality parameters such as hardness, maturity, sugar, water contents and so on, and estimates fruit quality by these parameters (Rongchao et al., 2007\& Wenchan, 2007). Under the effect of electric field, there is really a certain relevance between electric and textrue parameters for Lingwu Long-Jujube; At the same time, electrical characteristics provide a possibility of nondestructive testing for Lingwu jujube quality.

In this paper, the electrical parameters of Lingwu long-Jujube picked and stored at room temperature were measured dynamically in the scope of $3.16 \mathrm{kHz}$ to $50.12 \mathrm{kHz}$ to select the sensitive electrical parameters and appropriate test frequency, sensitive electric parameters trend along with storage periods studied, correlation between electrical parameters and texture parameters explored, in order to seek more rapid, convenient, and practical nondestructive identification methods for jujube texture quality evaluation, and lay the foundation for the effective implementation of the Lingwu Long-Jujube quality nondestructive testing technology.

\section{MATERIALS AND METHODS}

\subsection{Materials}

Testing samples: Non-disease and non-destruction Lingwu Long-Jujubes with consistent sizes, color, length were selected. The selected jujubes had $42 \pm 2 \mathrm{~mm}$ long diameter of axle, $30 \pm 2 \mathrm{~mm}$ short diameter of axle, a little color with green, 4/5 matured, and no pedicels. The jujubes were picked from Daquan forest farm in Lingwu, Ningxia, and transported to the lab at the same day, and stored at room temperature $\left(20 \pm 2^{\circ} \mathrm{C}\right)$ for use.

\subsection{Testing methods}

\subsubsection{Electrical parameters measurement}

The parameters were measured using 3532-50 LCR meter, HIOKI, connected to the computer through RS232 interface and operated by instrument software. Two rectangle parallel aluminum plate electrodes in $3 \mathrm{~mm}$ thickness were employed. Testing conditions: environmental temperature $20 \pm 2^{\circ} \mathrm{C}$; testing voltage $1 \mathrm{~V}$.

The testing jujubes were laid between the two parallel plate electrodes connected to the LCR meter through a wire. Each group with eight jujubes was tested, and the testing repeated for three times. The complex impedance $\mathrm{Z}$, parallel equivalent inductance $\mathrm{Lp}$ and reactance $\mathrm{X}$ of Lingwu LongJujube were measured at 25 frequency points: $10^{3.5}$, $10^{3.55}, 10^{3.6}, 10^{3.65}, 10^{3.7}, 10^{3.75}, 10^{3.8}, 10^{3.85}, 10^{3.9}$, $10^{3.95}, 10^{4}, 10^{4.05}, 10^{4.1}, 10^{4.15}, 10^{4.2}, 10^{4.25}, 10^{4.3}$, $10^{4.35}, 10^{4.4}, 10^{4.45}, 10^{4.5}, 10^{4.55}, 10^{4.6}, 10^{4.65}, 10^{4.7} \mathrm{~Hz}$ in the scope of $10^{3.5} \sim 10^{4.7} \mathrm{~Hz}(3.16 \mathrm{kHz} \sim 50.12 \mathrm{kHz})$.

\subsubsection{Texture parameters measurement}

Texture properties were measured by physical property analyzer TA. XT. Plus, SMS. 24 jujubes randomly taken from fruit cases were measured three times and their average value was calculated. The Long-Jujubes were laid on the platform of the property analyzer and measured by $\mathrm{P} / 2 \mathrm{n}$ probe.

The pre-testing rate, testing rate, and post-testing rate were all set as $1 \mathrm{~mm} / \mathrm{s}$. The trigger force value was $5 \mathrm{~g}$, puncture depth $5 \mathrm{~mm}$. Texture parameters such as cracking depth $(\mathrm{mm})$, pericarp strength $(\mathrm{g})$, brittleness $(\mathrm{g} / \mathrm{sec})$, pulp firmness $(\mathrm{g})$, and hardness (g) were obtained from the texture characteristic curve(Rongchao, Wen \& Weixiong et al., 2007).

\section{RESULTS AND DISCUSSION}

\subsection{The texture property changes of Lingwu Long- Jujube}

Long jujube texture property test curve was showed in Fig.1. Pericarp strength (g) was the force needed when the pericarp was broken. The first peak (anchor 2) value was pericarp strength. The puncturing distance was the pericarp broken depth. The ratio of the first peak value to the puncturing distance was pericarp brittleness $\left(\mathrm{g} \cdot \mathrm{sec}^{-1}\right)$. The average force value of anchor 3 and 4 was pulp firmness $(\mathrm{g})$. The peak value of anchor 4 was the greatest degrees of hardness(Camps et al.,2005) .

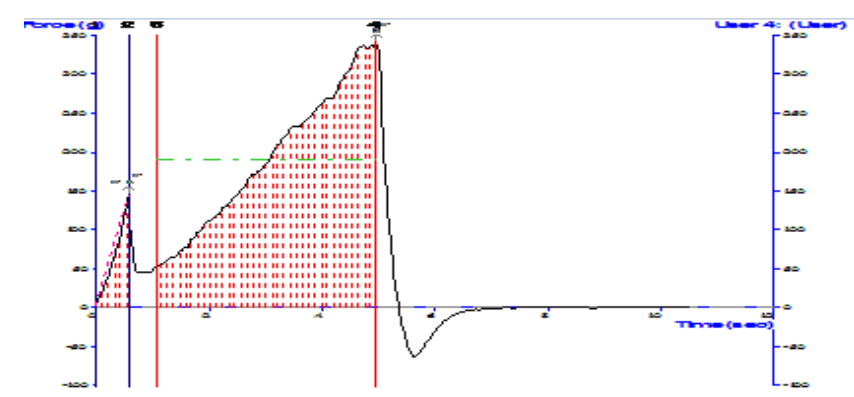

Fig 1.The texture characteristic curve

Table 1 Texture parameters' changes of Long jujube during storage

\begin{tabular}{c|c|c|c|c|c|}
\hline $\begin{array}{c}\text { Storage } \\
\text { time/d }\end{array}$ & $\begin{array}{c}\text { Bursting } \\
\text { depth } / \mathrm{mm}\end{array}$ & $\begin{array}{c}\text { Pericarp } \\
\text { strength } / \mathrm{g}\end{array}$ & $\begin{array}{c}\text { Brittleness } \\
\mathrm{g} / \mathrm{sec}\end{array}$ & $\begin{array}{c}\text { Pulp firmness } \\
/ \mathrm{g}\end{array}$ & Hardness/g \\
\hline 1 & $0.337 \pm 0.48$ & $116.26 \pm 13.90$ & $460.54 \pm 70.27$ & $266.37 \pm 23.93$ & $466.448 \pm 78.62$ \\
\hline 2 & $0.264 \pm 0.05$ & $115.26 \pm 15.38$ & $427.54 \pm 67.59$ & $254.956 \pm 26.83$ & $472.895 \pm 48.83$ \\
\hline 3 & $0.373 \pm 0.05$ & $125.72 \pm 16.89$ & $326.62 \pm 55.52$ & $226.88 \pm 22.96$ & $420.84 \pm 37.71$ \\
\hline 4 & $0.456 \pm 0.08$ & $122.76 \pm 15.26$ & $269.76 \pm 54.13$ & $202.31 \pm 19.81$ & $367.66 \pm 34.01$ \\
\hline 5 & $0.596 \pm 0.09$ & $124.92 \pm 18.01$ & $210.73 \pm 37.21$ & $171.62 \pm 19.64$ & $311.30 \pm 33.10$ \\
\hline 6 & $0.597 \pm 0.13$ & $127.18 \pm 17.48$ & $218.35 \pm 54.05$ & $168.43 \pm 25.69$ & $299.18 \pm 48.21$ \\
\hline 7 & $0.675 \pm 0.11$ & $132.44 \pm 16.62$ & $199.14 \pm 47.49$ & $159.02 \pm 18.75$ & $280.57 \pm 34.42$ \\
\hline
\end{tabular}

Note: average value \pm SD $(n=24)$

Table 1 showed the variation value of long jujube texture parameters during storage. The pericarp strength is not only related to jujube tissueal structure, but also to its water content. Postharvest long jujube pericarp strength will become strong along with the increasing of maturity and the loss of moisture. Brittleness is an important index reflecting 
fresh long jujube's resistance to vibration and abrasion. It is usually associated with stress relaxation. To some extent, it reflects long jujube's chewiness. Brittleness decreases gradually with the extension of storage time duo to the dehydration in long jujube's internal tissue. Pulp firmness is the compaction degree of pulp tissue. Brittleness and hardness can reflect pulp firmness(Wenchuan,2007). The greater the hardness, the greater the brittleness of jujube, and the tighter the pulp tissue

\subsection{The electrical property parameters'variation of jujube during storage}

The variation of long jujube's complex impedance $(\mathrm{Z})$, reactance $(\mathrm{X})$ and parallel equivalent inductance (Lp) was tested and analyzed in the selected frequency range from $3.16 \mathrm{kHz}$ to $50.12 \mathrm{kHz}$ (that is from $10^{3.5}$ to $\left.10^{4.7} \mathrm{~Hz}\right)$. Results were shown in Figure 2. As the test frequency increasing, the value of $\mathrm{Z}$, $X$, Lp decreased continuously. The higher the frequency, the smaller the variation of the value of $\mathrm{Z}, \mathrm{X}$ and Lp. As the storage time extending, the value of $Z, X, L p$ increased continuously. The higher the frequency, the smaller the increasing range.

In the same frequency range, the values of $\mathrm{Z}, \mathrm{X}$, and Lp increased slowly with the extension of storage time. Comparisons found, the effect of frequency was greater than that of storage time on Z, $X, L p$ values. And at the same frequency, the values of Z, X, Lp had a strong positive linear correlation with storage time $\left(R^{2}>0.67\right)$. In Table 2 , the fitting equation showed a good correlation between $\mathrm{Z}, \mathrm{X}$, $\mathrm{Lp}$ and testing frequency. In Table 3, regression equation of the electrical parameters and storage time under the frequency of $3.16 \mathrm{kHz}$ showed the correlation between $\mathrm{Z}, \mathrm{X}, \mathrm{Lp}$ and storage time $\mathrm{t}$ is significant or extremely significant.

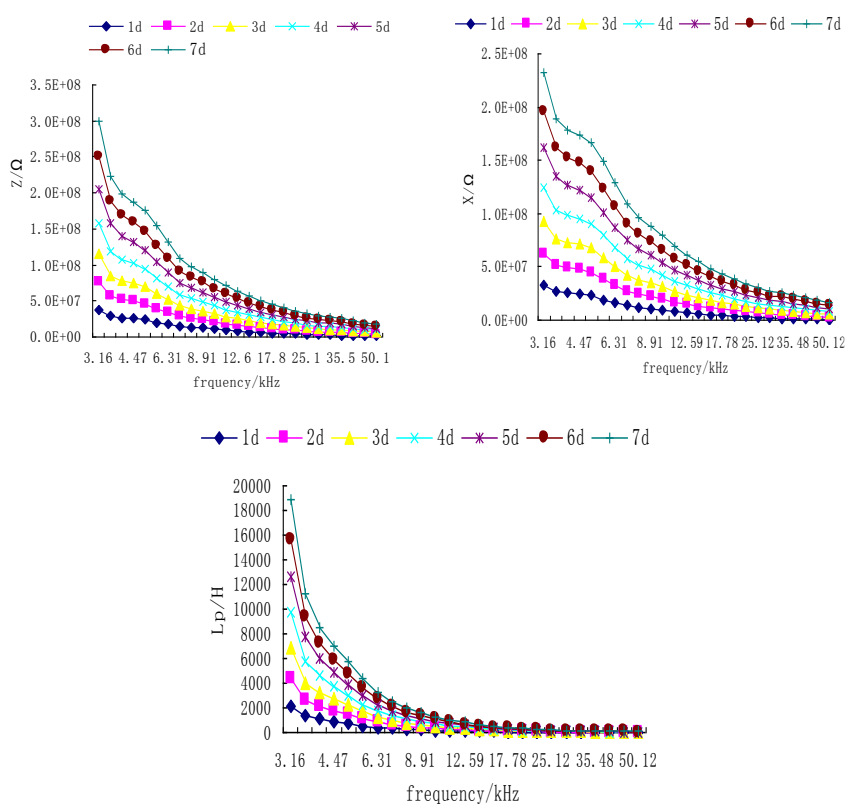

Fig 2. The curve of $\mathrm{Z}, \mathrm{X}$, and $\mathrm{Lp}$ with testing frequency changes
Table 2 The correlation between the electrical parameters of long jujube and the testing frequency

\begin{tabular}{|c|c|c|c|}
\hline Storage timed & $Z / \Omega$ & $\mathrm{X} / \Omega$ & $\mathrm{Lp} / \mathrm{H}$ \\
\hline 1 & $\begin{array}{c}\mathrm{y}_{2}=3 \times 10^{0} \mathrm{e} \mathrm{e}^{-0.0665 \mathrm{f}} \\
\mathrm{R}^{2}=0.9112\end{array}$ & $\begin{array}{l}y_{x}=3 \times 10^{0} 7.0 .0888 \\
R^{2}=0.9612\end{array}$ & $\begin{array}{l}y_{L p}=735.97 e^{0.0912} \\
R^{2}=0.8219\end{array}$ \\
\hline 3 & $\begin{array}{l}y_{2}=2 \times 10^{00} 2.003365 \\
R^{2}=0.5333\end{array}$ & $\begin{array}{l}y_{x}=2 \times 10^{70} e^{-0.05198} \\
R^{2}=0.8747\end{array}$ & $\begin{array}{l}\mathrm{YID}_{\mathrm{Ip}}=834.94 \mathrm{e} \\
\mathrm{R}^{2}=0.112828\end{array}$ \\
\hline 5 & $\begin{array}{l}y_{2}=3 \times 10^{07} e^{.005322} \\
R^{2}=0.832\end{array}$ & $\begin{array}{l}y_{x}=2 \times 10^{0} e^{-0.0528 f} \\
R^{2}=0.863\end{array}$ & $\begin{array}{l}\mathrm{y}_{L_{p}}=963.58 \mathrm{e} \mathrm{e}^{-01439 \mathrm{f}} \\
\mathrm{R}^{2}=0.5506\end{array}$ \\
\hline 7 & $\begin{array}{l}\mathrm{yz}=3 \times 10^{07} e^{-0.054 \mathrm{f}} \\
\mathrm{R}^{2}=0.8477\end{array}$ & $\begin{array}{l}y_{d}=3 \times 10^{07} \mathrm{e}^{-0.0522 \mathrm{f}} \\
\mathrm{R}^{2}=0.8723\end{array}$ & $\begin{array}{l}\mathrm{y}_{L_{p}}=1018.2 \mathrm{e} \\
\mathrm{R}^{2}=0.11454 \mathrm{f}\end{array}$ \\
\hline
\end{tabular}

Table 3 The regression equation of jujube electrical parameters and storage time at room temperature

\begin{tabular}{|c|c|}
\hline Regression equation & Determination coefficient \\
\hline$y_{2}=825436 \mathrm{t}+2 \times 10^{7} \mathrm{~T}$ & $\mathrm{R}^{2}=0.8322$ \\
\hline $\mathrm{y}_{\mathrm{x}}=635242 \mathrm{t}+2 \times 10^{07} \mathrm{P}$ & $\mathrm{R}^{2}=0.6784$ \\
\hline $\mathrm{y}_{\mathrm{Lp}}=35.075 \mathrm{t}+679.04$ & $\mathrm{R}^{2}=0.9219$ \\
\hline
\end{tabular}

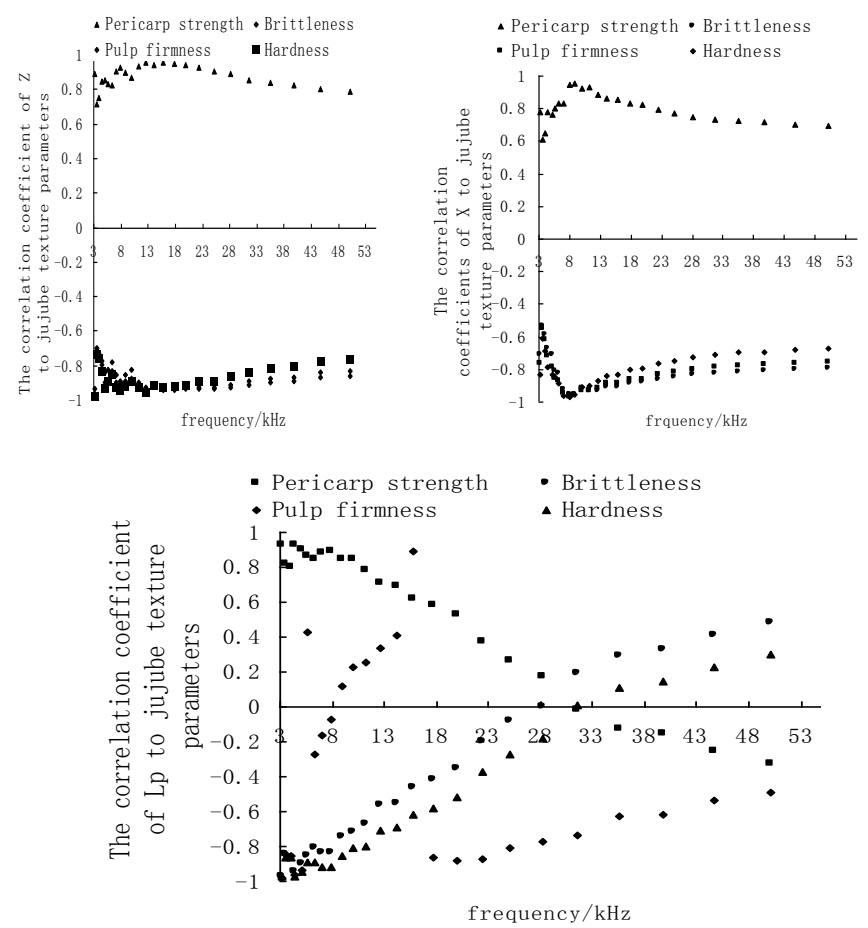

Fig 3.The correlation coefficients of $\mathrm{Z}, \mathrm{X}$ and $\mathrm{Lp}$ to jujube texture parameters

\subsection{Appropriate testing frequency selection for Lingwu long-jujube}

Chart 3 showed the correlation coefficients between $\mathrm{Z}, \mathrm{X}$, and Lp, and pericarp strength, brittleness, pulp firmness at 25 frequency points in the range of $3.16 \mathrm{kHz} 50.12 \mathrm{kHz}$ during jujube storage at room temperature. There is a good correlation between $\mathrm{Z}$, $\mathrm{X}$ and pericarp strength, brittleness, pulp firmness and hardness in this testing frequency range. As the testing frequencies increase, the correlation coefficients of $\mathrm{Lp}$ to those four texture parameters decrease continuously. 
At $3.16 \mathrm{kHz}$, correlation coefficients of $\mathrm{Z}, \mathrm{X}$, and Lp are $0.8937,0.78,0.92$ to pericarp strength respectively, $-0.9364,-0.71,-0.98$ to brittleness separately, and $-0.9667,-0.7648,-0.98359$ to pulp firmness. It was found that the values of $\mathrm{Z}, \mathrm{X}$, and $\mathrm{Lp}$ at $3.16 \mathrm{kHz}$ are significantly different from those at other frequencies by variance analysis $(\mathrm{P}<0.05)$. Therefore, $3.16 \mathrm{kHz}$ is the appropriate frequency for jujube electrical properties testing in the selected measurement frequency ranges.

Table 4 Correlation analysis of long jujube electrical physical parameters and texture parameters

\begin{tabular}{|c|c|c|c|}
\hline \multirow{2}{*}{ Texture parameters } & \multicolumn{3}{|c|}{ Correlation coefficient } \\
\cline { 2 - 4 } & $Z$ & $X$ & Lp \\
\hline Pericarp strength & $0.893 T^{*}$ & 0.78186 & $0.92288^{\circ}$ \\
\hline Brittleness & $-0.9364^{\circ}$ & -0.71076 & $-0.97675^{\circ}$ \\
\hline Pulp firmness & $-0.9667^{\circ}$ & $-0.7648^{\circ}$ & $-0.98359^{\circ}$ \\
\hline Hardness & $-0.97742^{\circ}$ & $-0.82935^{\circ}$ & $-0.97546^{\circ}$ \\
\hline
\end{tabular}

Table 5 The regression equations of long jujube physical parameters and texture parameters during room temperature storage

\begin{tabular}{|c|c|c|}
\hline Brittleness & $Y_{s x}=1008.03827 .0 .00000863 \times 1+0.00000587 \times 2-0.19713 \times 3$ & $R^{2}=0.9601$ \\
\hline Pulp finmesst & $Y_{n n z x 又}=534.81417-0.00000503 \times 1+0.00000158 \times 2-0.06120 x 3$ & $\mathrm{R}^{2}=0.9805$ \\
\hline Hardness & $Y_{g s}=1046.02835-0.0000066 \times 1-0.00000277 \times 2-0.11047 \times 3$ & $R^{2}=0.9790$ \\
\hline
\end{tabular}

Note: $x 1, x 2, x 3$ represent $Z, X$, and Lp separately.

\subsection{Correlation analysis of long jujube electrical parameters and texture parameters}

Table 4 showed the results of correlation analysis between electrical parameters and texture parameters at the appropriate $3.16 \mathrm{kHz}$ testing frequency. Electrical parameters have a good correlation with texture parameters. $\mathrm{Z}$ has a strong correlation with pericarp strength, brittleness, and pulp firmness $(\mathrm{R}=0.8937,-0.9364,-0.9667$ respectively). Lp has a strong correlation with pericarp strength, brittleness, and pulp firmness also $(\mathrm{R}=0.92288,-0.97675$, -0.98359 respectively). The correlation reaches extreme significant level $(\mathrm{P}<0.01)$. $\mathrm{X}$ has a good correlation with pericarp strength and pulp firmness $(\mathrm{R}=0.78186$, -0.7648), the correlation reaches significant level $(\mathrm{P}<0.05)$. Regression analysis of texture parameters and electrical parameters at the appropriate testing frequency has been done based on the principle of minimum square method. Regression equation was showed in Table 5. It is found by polynomial regression analysis that jujube pericarp strength has no correlation with $\mathrm{Z}, \mathrm{X}$, and Lp ( $P>0.05)$. Brittleness and pulp firmness have significant correlation with $\mathrm{Z}, \mathrm{X}$, and $\mathrm{Lp}(\mathrm{P}<0.05)$; pulp firmness has extreme significant correlation with $\mathrm{Z}, \mathrm{X}$, and $\mathrm{Lp}(\mathrm{P}<0.01)$. By measuring $\mathrm{Z}, \mathrm{X}, \mathrm{Lp}$, the brittleness, pulp firmness and hardness of long jujube fruit can be indirectly obtained through the regression equation. Therefore, it provides a rapid, simple and reliable non-destruction testing method.

\section{DISCUSSION}

\subsection{The frequency dependence of electrical parameters of jujube at room temperature storage}

Besides the tissue, the composition, structure, and status of the measured object have some influence on its electrical characteristics, the frequency of the electromagnetic field also directly affect the electrical properties of the tested materials (Lite,2009; Xinhua, Wenchuan \& Kangquan, 2004; Vankatesh \& Raghavan,2004). This study showed in the frequency range of $3.16 \mathrm{kHz} 50.12 \mathrm{kHz}$, jujube's $Z, X$, and Lp have a similar decreasing trend which has a strong exponential regression relationship with testing frequencies. In low frequencies jujube extracellular ionic conductivity increases the values of $\mathrm{Z}, \mathrm{X}, \mathrm{Lp}$. As the frequency increases continuously, it changes jujube dielectric polarization's mode and degree in electro-magnetic field, and leads to electrical parameters continuous decrease.

\subsection{Storage time-varying of electrical parameters of jujube}

Under natural room temperature, jujube storage time is shorter and up to 7 days or so at most. Storage time and maturation process affect long-jujube electrical properties. As the storage time extending, jujube water content decreases, extracellular fluid gets less and less. Due to the weakening of ionic conduction, the Z, X, Lp of jujube fruit increase with the extension of storage time. Under the same frequency, Z, X, Lp values present a strongly positive linear correlation with storage time $\left(\mathrm{R}^{2}>0.67\right)$.

\subsection{Storage time-varying of texture parameters of jujube}

Texture property testing results obtained from whole fruit puncture method showed that the pericarp strength and broken depth increase but brittleness, pulp firmness and hardness decrease gradually with the storage time extending. This is because that at the beginning of jujube storage, newly picked jujube contains large amounts of water in its tissue, and also protopectin combines tightly to cell-wall, pulp tissue packs closely. Thus jujube pulp brittleness is high as well as the hardness. Protopectin is under continuous hydrolysis and the water content in longjujube tissue decreases. This makes jujube fruit pericarp ruffle. In addition, cellulose and lignin in 
jujube pericarp will increase, the pericarp thickness will become thicken, and lead to pericarp strength increasing.

\subsection{The correlation between electrical parameters and texture parameters}

$3.16 \mathrm{kHz}$ is the aappropriate frequency for longjujube electrical parameters testing. Electrical parameters Z, X, and Lp have a high correlation with texture parameters at this frequency. Regression equation establishes the relation between electrical parameters and textrue parameters. Electrical parameters' measurement is rapid, sensitive, and easy to operate. Jujube fruit texture parameters can be indirectly obtained through rapid long-jujube electrical parameters' testing. It has very important values in the jujube internal quality testing, processing and storing, especially has a broad prospects in fruit grading.

\section{ACKNOWLEDGEMENT}

The authors are grateful to the Chinese national natural science fund for its financial support for this research.

\section{REFERENCES}

[1] Camps C,Guilermin P,Mauget J C,Bertrand D.Data analysis of penetrometric force/displacement curves for the characterization of whole apple fruits. Journal of Texture Studies, 2005, 36: 387-401.

[2] Feng Z. Research progress in mechanism of ripening and softening of stone fruit. Acta agriculturae Jiangxi, 2011, 23(4):62-65.

[3] Lite L. physical character of food. Beijing:Chinese Agricultural Press, 2001 :96-100.
[4] Qinghua M, Guixi W, Lisong L. Establishment of the detecting method on the fruit texture of Dongzao by puncture test. Scientia Agricultura Sinica, 2011, 44(6):1210-1217.

[5] Rongchao M, Wen Q, Weixiong W, etal. Review of research advances in non-destructive quality evaluation technology of food by dielectric property. Transactions of the CSAE, 2007, 23(5):278-783.

[6] Venkatesh, M.S., Raghavan, G.S.V.An overview of microwave processing and dielectric properties of agrifood materials. Canadian Biosystems Engineering, 2004, 88(1): 1-18.

[7] Wenchuan G. Review of dielectric properties of fruits and vegetables. Transactions of the CSAE, 2007, 23(5):284289.

[8] Xinhua Z, Wenchuan G, Kangquan G. Influence of electric excitation signal on electric parameters of fruits. Jour. of Northwest Sci-Tech Univ. of Agri. and For.(Nat. Sci. Ed.), 2004, 32(11):125-128.

[9] Yaping L, Hongbo L. Review on the application of Texture Analyzer and TPA in the assessment for fruits and vegetables. J.JIANGXI AGRIC UNIV.(NaturalScience Edition), 2010, 30(2):188-189.

\section{AUTHOR INTRODUCTION:}

Zhou Shiping (1989-), male, Ningxia Guyuanese, Current Master Students in Ningxia University, food machinery and automation control research area; contact phone number: 18095163134

E-mail: zhouyuxin.2008@163.com

Corresponding author: Zhang Haihong (1967-), female, Ningxia Yinchuanese, professor of Ningxia University, Master's Supervisor, mainly engaged in research of Physical Properties of Foods; contact phone number: 1395277851

E-mail: nxdwjyxx@126.com 